\section{So rechnen Sie ab Juli die Videosprechstunde ab}

\begin{abstract}
_ KBV und GKV-Spitzenverband haben sich über die Rahmenbedingungen der Videosprechstunde gemäß $₫ 291 \mathrm{~g}$ Abs. 4 SGB V geeinigt. Für die benötigte Hardware wie auch für die elektronische Datenübertragung wurden Standards definiert. Gleichzeitig wurde auch die Honorierung festgezurrt.

Vor der ersten Videosprechstunde muss der Patient schriftlich einwilligen. Der Arzt muss sich bei einem geeigneten
\end{abstract}

Videodienst registrieren. Patienten müssen sich dort ohne Account anmelden können, ihr Name soll für den Arzt aber erkennbar sein. Der Zugang darf nur zum Kontakt mit dem initiierenden Arzt führen und muss zeitlich auf höchstens einen Monat befristet sein. Die Übertragung der Videosprechstunde muss über eine sogenannte Peer-to-Peer-Verbindung erfolgen, darf also nicht über einen zentralen Server laufen. Sämtliche In-

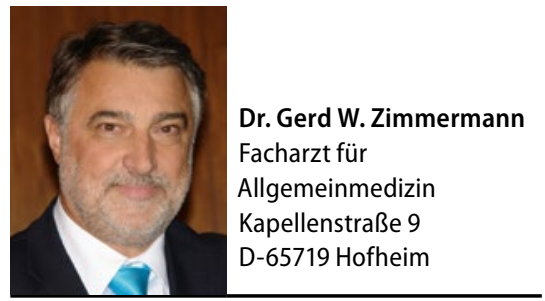

halte müssen während des gesamten Übertragungsprozesses verschlüsselt werden.

\section{MMW-KOMMENTAR}

Eine Videosprechstunde gilt im EBM als "anderer Arzt-Patienten-Kontakt" nach Punkt 4.3.1 der Allgemeinen Bestimmungen. Die Leistung kann im hausärztlichen Bereich nach Nr. 01450 EBM als Zuschlag zu den altersgestaffelten Versichertenpauschalen nach Nr. 03000 oder der Kontaktpauschale nach $\mathrm{Nr} .01435$ berechnet werden. Sie wird im Rahmen der budgetierten Gesamtvergütung mit 137 Punkten (14,43 Euro) vergütet, wobei für jede Praxis ein Höchstwert von 2.393 Punkten im Quartal gilt. Bei Gebührenordnungspositionen, deren Berechnung mindestens zwei persönliche Arzt-Patienten-Kontakte im Behandlungsfall voraussetzt z.B. bei der Chronikerpauschale nach Nr. 03221 -, zählt eine Videosprechstunde als einer dieser Kontakte. Die Abrechnung der neuen Leistung ist ab dem 1. Juli 2017 möglich.

\title{
Alle Notfallleistungen künftig ohne Budget?
}

_ Aktuell wird das Honorar für Leistungen des organisierten Not- bzw. Bereitschaftsdienstes ohne Abzüge an die Ärzte ausgezahlt, noch bevor die morbiditätsorientierte Gesamtvergütung (MGV) in einen haus- und einen fachärztlichen Anteil aufgeteilt wird. Dieser Grundbetrag „Ärztlicher Bereitschaftsdienst“ muss wegen einer Gesetzesänderung im $\$ 87 \mathrm{~b}$ Abs. 1 SGB V nun angepasst werden. Der neu eingefügte Satz 3 sieht nämlich die unquotierte Vergütung aller Leistungen im Notfall und im Notdienst aus einem vor der Trennung für die Versorgungsbereiche gebildeten Honorarvolumen vor. Es kommen also alle Notfallleistungen außerhalb des organisierten Notdienstes hinzu.

\section{MMW-KOMMENTAR}

In der Gesetzesbegründung wird zur Abgrenzung des Notfalls auf die Rechtsprechung des Bundessozialgerichts zu § 76 Abs. 1 Satz 2 SGB V verwiesen. Die höchsten Richter haben nämlich auch Nicht-Vertragsärzten vorgegeben, dass sie einen Patienten in einem Notfall aus medizinischen Gründen umgehend behandeln müssen. Dies ist nach Auffassung der Richter vor allem dann der Fall, wenn ohne sofortige Behandlung Gefahren für Leib und Leben entstehen oder heftige Schmerzen unzumutbar lange andauern würden. 